\title{
Ortsaufgelöste in-situ Beladungsdiagnose von Diesel-Partikelfiltern
}

\author{
Gunter Hagen, Andreas Piontkowski, Andreas Müller, Dieter Brüggemann, Ralf Moos \\ Bayreuth Engine Research Center (BERC), Universität Bayreuth, 95440 Bayreuth
}

\section{Zusammenfassung}

Der Einsatz von Partikelfiltern in dieselbetriebenen Fahrzeugen ist notwendig, um die strengen gesetzlichen Emissionsgrenzwerte einzuhalten. Dabei wird im diskontinuierlichen Betrieb ein im Abgasstrang eingebautes Filterelement mit Ruß beladen und mit erhöhtem Kraftstoffaufwand durch Erhöhung der Abgastemperatur wieder regeneriert. Für den effizienten Betrieb eines solchen Systems, also z.B. zur Bestimmung eines geeigneten Regenerationszeitpunkts, ist es wünschenswert, die im Filter enthaltene Rußmenge und deren Verteilung genau zu kennen. Durch Kontaktierung einzelner Kanäle innerhalb des Filters konnte eine Möglichkeit gefunden werden, die Ablagerung von Ruß direkt zu messen. Rußpartikel bilden leitfähige Pfade zwischen den elektrisch kontaktierten Filterwänden. Der gemessene Widerstand hängt somit direkt von der Rußmenge im Filter ab. In einer speziellen Anordnung konnte damit die örtliche Verteilung des Rußes innerhalb eines Partikelfilers während der Beladung am Motorprüfstand bestimmt werden.

\section{Motivation}

Ohne den Einsatz von Diesel-Partikelfiltern (DPF) können die strengen Abgas-Grenzwerte für neue Fahrzeuge nicht erreicht werden. Gesundheitsgefährdende Rußpartikel, die bei der motorischen Verbrennung entstehen, werden in keramischen Filterelementen gesammelt und zu gegebener Zeit durch eine spezielle Betriebsstrategie verbrannt. Ein DPF besteht aus einem keramischen Wabenkörper mit wechselseitig verschlossenen Kanälen. Das Abgas muss durch die porösen Filterwände strömen; Rußpartikel lagern sich dabei zuerst in den Filterwänden und dann als Filterkuchen in den eingangsseitig geöffneten Kanälen ab. Ist eine bestimmte Rußmenge erreicht (das Filter-Volumen also „erschöpft“), muss der Filter regeneriert werden. Dies geschieht häufig durch Nacheinspritzung von Kraftstoff und Erhöhung der Abgastemperatur, wodurch der gesammelte Ruß verbrannt wird. Der Regenerationszeitpunkt wird derzeit über eine Differenzdruckmessung über dem Filter ermittelt. Der Differenzdruck gibt jedoch lediglich ein indirektes Maß der Rußmenge im Filter an [1].

Für einen effizienten Betrieb solcher Systeme wäre die genaue Kenntnis des Beladungszustandes des Filters wünschenswert. In der Literatur wird dazu beispielsweise ein Hochfrequenz-Messverfahren vorgeschlagen [2]. Dieses System wurde schon erfolgreich im Bereich der Zustandsdiagnose von Drei-Wege-Katalysatoren erprobt [3-4]. Erste Versuche zeigen auch die Möglichkeit einer Übertragung dieses Verfahrens auf den Zustand der Ammonik-Beladung von SCR-Katalysatoren [5]. In Bezug auf die Partikelfilterdiagnose liefert dieses Verfahren einen integralen Wert der tatsächlichen Rußbeladung im Filter. Der DPF mit seinem Metallgehäuse wird als Hohlleiterresonator angesehen. Die Dämpfung oder Resonanzverschiebung eines eingekoppelten Signals im GHz-Bereich hängt direkt von der Beladung des Filters mit leitfähigen Rußpartikeln ab.

Für eine Regenerationsstrategie, die außerdem den Schutz der Bauteile (Überhitzung) im Blick hat, wäre zusätzlich die Kenntnis der Rußverteilung im Filter eine notwendige Größe. Im vorliegenden Beitrag konnte durch die Kontaktierung einzelner Kanäle und die Messung der Rußablagerung an verschiedenen Stellen im Filter eine Möglichkeit gefunden werden, ortsaufgelöste Informationen über die Beladung eines Partikelfilters zu erhalten. 


\section{Experimentelles}

Um die Beladung des Filters direkt messen zu können, wurden einzelne Kanäle bzw. Wände eines handelüblichen unbeschichteten DPF-Substrats (Wabenkörper aus Aluminiumtitanat) kontaktiert. Dazu wurde mittels einer Kanüle eine bestimmte Menge verdünnter Goldpaste von der Ausgangsseite her in einzelne Kanäle eingebracht. Die Goldpaste fließt dabei in die porösen Filterwände. Die Auslass-Kanäle werden dabei nicht verschlossen, es entsteht aber ein Kurzschluss zwischen den benachbarten Wänden. Nach einer Temperaturbehandlung liegen somit elektrisch leitfähige Wandbereiche vor, deren laterale Ausdehnung von der Menge an eingebrachter Paste abhängt. Bei allen Kontakten wurde jeweils dieselbe Pastenmenge verwendet. Zwischen den kontaktierten Stellen befinden sich die Einlass-Kanäle, in denen der elektrische Kontakt über den abgeschiedenen Ruß zu Stande kommt. Bild 1 verdeutlicht schematisch den Aufbau der Messanordnung.

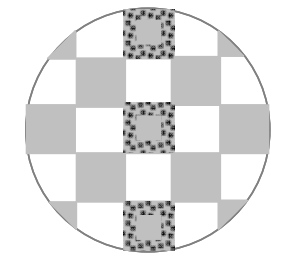

a)
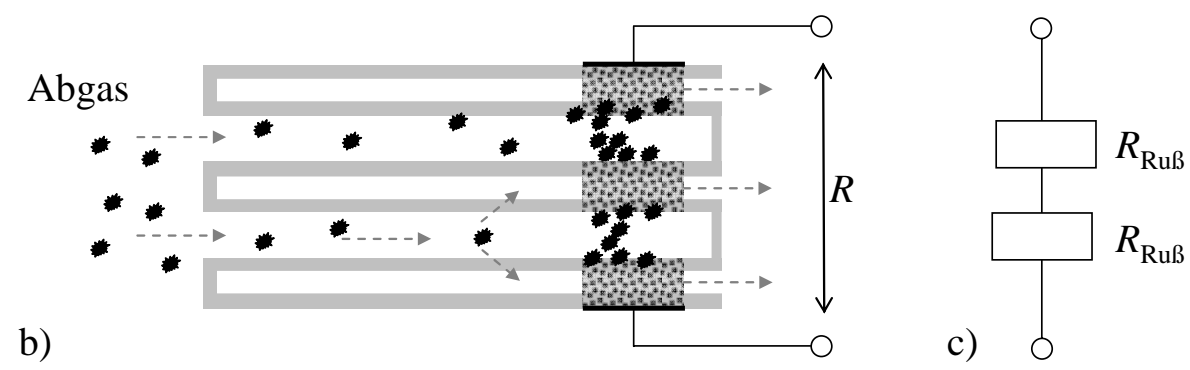

Bild 1 Schematische Ansicht eines DPF mit wechselseitig verschlossenen Kanälen: frontale Ansicht (a) und Querschnittsdarstellung (b) zur Widerstandsmessung über infiltrierte Kanäle, Rußablagerungen bilden leitfähige Pfade zwischen den Kontakten aus $\left(=R_{\text {Rus }}\right)$. Der Messwiderstand setzt sich aus den kontaktierten Kanälen als Sereienschaltung zusammen (c).

Während der Rußablagerung im DPF wird nach Erreichen einer Perkolation durch Rußpartikel ein messbarer Widerstand erwartet, der im weiteren Betrieb abnehmen sollte, da weitere Abscheidung zur Vergrößerung des „Leiter“querschnitts führt. Der sinkende Widerstand soll also mit der steigenden Rußmenge im Partikelfilter einhergehen.

Um die Rußbeladung im DPF lokal aufgelöst vermessen zu können, wurde ein spezieller Filter präpariert: Über die laterale Achse des Filters wurden vier Messstellen ( $R_{1}=$ eingangsseitig, $R_{2}, R_{3}, R_{4}=$ ausgangsseitig) mit jeweils fünf offenen Kanälen (5 x $R_{\text {Ruß }}$ ) eingebracht (Bild 2).

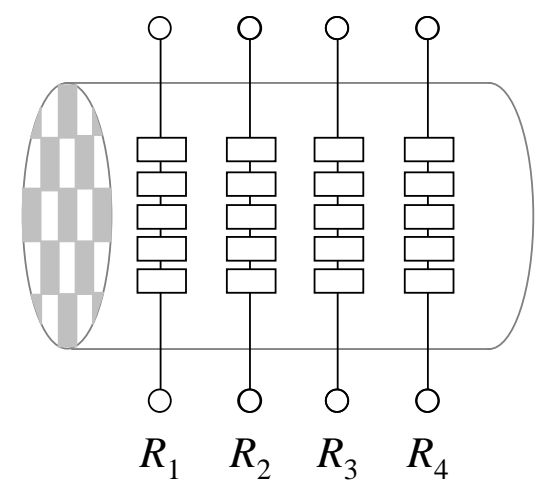

Bild 2 Verteilung der Messstellen zur ortsaufgelösten Messung der Rußbeladung eines Diesel-Partikel-Filters: $R_{1}$ (eingangsseitig), $R_{2}, R_{3}, R_{4}$ (ausgangsseitig). Jeder Widerstand stellt eine Serienschaltung über fünf Kanäle dar (5 x $\left.R_{\mathrm{RuB}}\right)$.

Der präparierte Partikelfilter wurde in den Abgasstrang eines 3,0 I TDI eingebaut und unter Realbedingungen untersucht. Die Beladung erfolgte bei konstantem Betrieb mit $1000 \mathrm{U} / \mathrm{min}$ unter $40 \%$ Last. Eine Regeneration wurde durch Drehzahlerhöhung unter 100 \% Last herbeigeführt. 


\section{Ergebnisse und Diskussion}

Die gemessenen Widerstandswerte am Partikelfilter-Aufbau während der Rußbeladung verhalten sich erwartungsgemäß. Man erkennt fallende Widerstände mit zunehmender Betriebszeit. Diese Ergebnisse ähneln stark dem Widerstandsverlauf eines einfachen Rußsensors im Abgasstrang [6]. In Bild 3 erkennt man deutlich die reproduzierbare Messung während dreier Beladungszyklen.

Die Verteilung der absoluten Widerstände ergibt (unter Annahme gleicher Kontaktgeometrien) ebenfalls ein nachvollziehbares Bild: Eingangsseitig $\left(R_{1}\right)$ treten die höchsten Widerstände auf, über die laterale Achse des Filters fallen die Widerstandswerte. Der Filter wird in diesem Fall also von hinten her beladen. Betrachtungen aus der Literatur bestätigen dieses Verhalten, wenngleich dieser Fall nicht verallgemeinerbar ist und stark von den Betriebsbedingungen abhängt [7].

Nach Erreichen eines bestimmten Beladungszustandes (hier erstmals nach ca. $1700 \mathrm{~s}$ ) wird eine Regeneration eingeleitet. Mit Beginn der Temperaturerhöhung fallen die Widerstandswerte (mit Ausnahme $R_{1}$ ) stark ab, was auf die verbesserte Rußleitfähigkeit bei erhöhter Temperatur zurückzuführen ist. Messungen an Rußsensoren [6], Messungen zur Koks-Beladung an Festbett-Katalysatoren [8] oder Arbeiten an Fulleren-Strukturen [9] bestätigen diese Annahme. Im Fall des Widerstands $R_{1}$ zeigt sich ein starker Anstieg mit Beginn der Regeneration. Möglicherweise wird der Ruß durch den erhöhten Abgasstrom bei hoher Drehzahl und Last in die hinteren Bereiche des DPF verschoben.

Nach Erreichen der Abbrand-Temperatur (ca. $600{ }^{\circ} \mathrm{C}$ ) steigen die Widerstandswerte aller Bereiche an und erreichen ein konstantes Niveau, was auf die Leitfähigkeit des Aluminiumtitanat schließen lässt. Ein weiterer Anstieg der Widerstände ist erkennbar, wenn die Abgastemperatur wieder sinkt und der Betriebspunkt für die Beladung eingestellt wird.

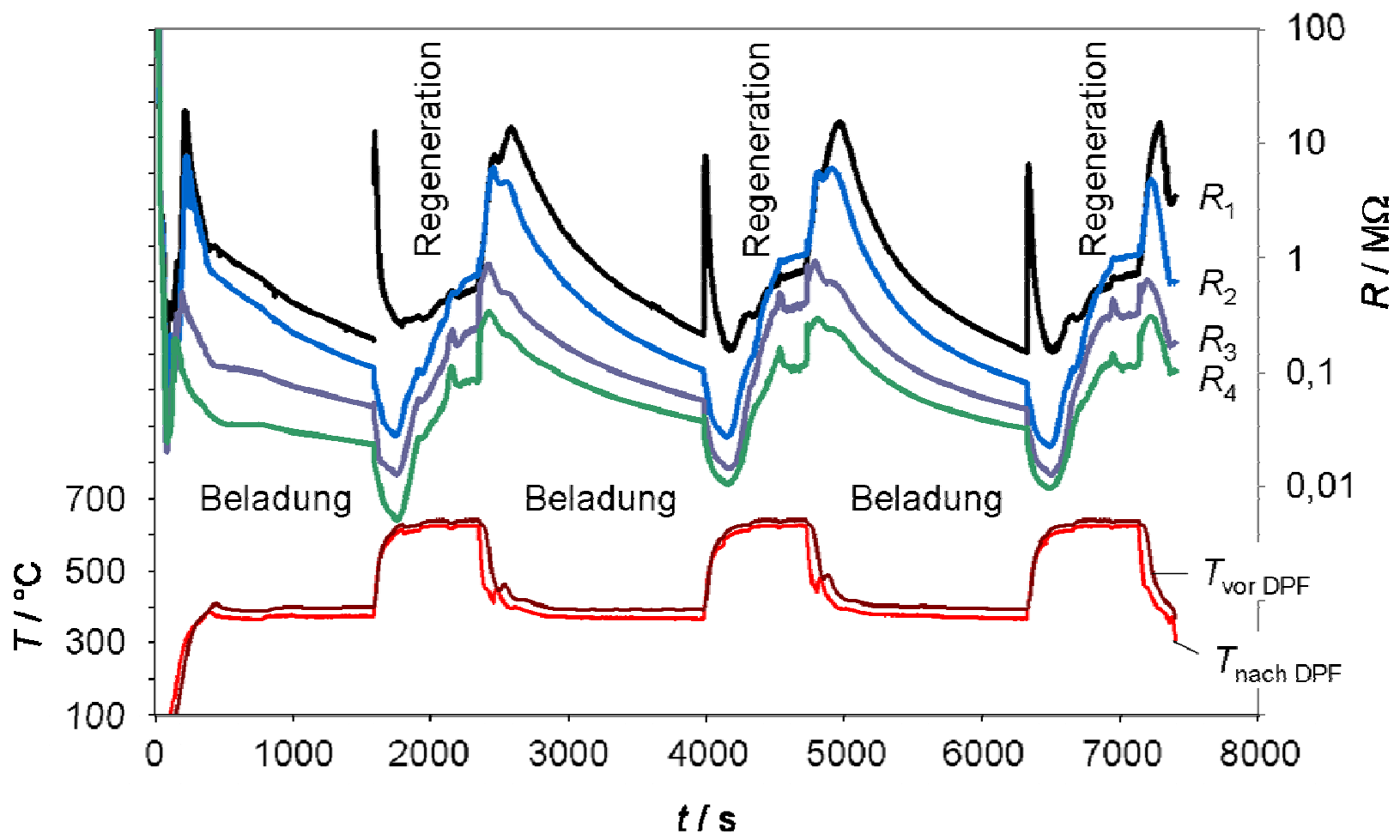

Bild 3 Messungen an einem direkt kontaktierten Partikelfilter im Realabgas eines 3,0 l TDI. 


\section{Zusammenfassung und Ausblick}

Mit der vorgestellten Methode, Kanäle im Inneren einen Partikelfilters zu kontaktieren, ist es möglich den Beladungszustand eines DPF direkt zu bestimmen. Die Kontaktierung und Messung an verteilten Stellen über der Längsachse des Filters erlaubt eine ortsaufgelöste Messung der Rußablagerungen.

Weitergehende Versuche sollen eine Korrelation zwischen den Signalen von Rußsensoren und der Filterbeladung herstellen, sowie die Temperaturabhängigkeit des Widerstandes und das Gegendruckverhalten des Filters beleuchten. Die Variation der Betriebsparameter ist ebenso erforderlich. Die Ergebnisse sind allerdings spezifisch bezüglich des Messaufbaus und der verwendeten Motorparameter. Die Vielzahl an Einflussfaktoren auf die Rußbildung [10] erlaubt keine pauschalen Aussagen. Allerdings zeigt sich, dass die vorgestellte Methode geeignet ist, die Vorgänge im Partikelfilter näher zu beleuchten und das Systemverständis zu fördern.

\section{Danksagung}

Für die finanzielle Unterstützung durch die Deutsche Forschungsgemeinschaft (DFG) unter MO 1060/6-2 sei herzlich gedankt.

\section{Literatur}

[1] M. V. Twigg, Progress and future challenges in controlling automotive exhaust gas emissions, App. Catal. Env. 70, S. 2-15, 2007

[2] G. Fischerauer, M. Förster, R. Moos, Sensing the Soot Load in Automotive Diesel Particulate Filters by Microwave Methods, Meas. Sci. Tech. 21, 035108, 2010

[3] R. Moos, M. Wedemann, M. Spörl, S. Reiß, G. Fischerauer, Direct Catalyst Monitoring by Electrical Means: An Overview on Promising Novel Principles, Top. Catal. 52, S. 2035-2040, 2009

[4] S. Reiß, M. Spörl, G. Hagen, G. Fischerauer, R. Moos, Combination of wirebound and microwave measurements for in-situ characterization of automotive three-way catalysts, IEEE Sensors Journal 11, S. 434-438, 2011

[5] S. Reiß, D. Schönauer, G. Hagen, G. Fischerauer, R. Moos, Monitoring the ammonia loading of zeolite-based ammonia SCR catalysts by a microwave method, Chem. Eng. Technol. 34, S. 791-796, 2011

[6] G. Hagen, C. Feistkorn, S. Wiegärtner, A. Heinrich, D. Brüggemann, R. Moos, Conductometric Soot Sensor for Automotive Exhausts: Initial Studies, Sensors 10, S. 1589-1598, 2010

[7] M. Schejbal, M. Marek, M. Kubicek, P. Koci, Modelling of diesel filters for particulates removal, Chem. Eng. J. 154, S. 219-230, 2009

[8] N. Müller, R. Moos, A. Jess, In situ monitoring of coke deposits during coking and regeneration of solid catalysts by electrical impedance-based sensors, Chem. Eng. Technol. 33, S. 103-112, 2010

[9] L. Dunne, A. Sarkar, H. Kroto, J. Munn, P. Kathirgamanathan,U. Heinen, J. Fernandez, J. Hare, D. Reid, A. Clark, "Electrical, magnetic and structural characterization of fullerene soots." J. Phys.: Condens. Matter 8, S. 2127-2141, 1996

[10] M. Maricq, Chemical characterization of particulate emissions from diesel engines: A review, Aerosol Science 38, S. 1079-1118, 2007 